\title{
ARTICLE
}

Pharmacology

\section{Daratumumab plus lenalidomide and dexamethasone in relapsed/ refractory multiple myeloma: extended follow-up of POLLUX, a randomized, open-label, phase 3 study}

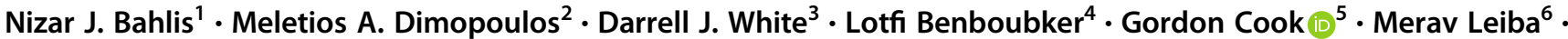

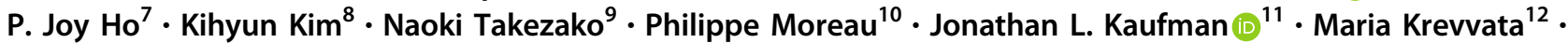 \\ Christopher Chiu ${ }^{12} \cdot$ Xiang Qin $^{12} \cdot$ Linda Okonkwo $^{13} \cdot$ Sonali Trivedi ${ }^{12} \cdot$ Jon Ukropec $^{14} \cdot \mathrm{Ming} \mathrm{Qi}^{12}$. \\ Jesus San-Miguel ${ }^{15}$
}

Received: 11 September 2019 / Revised: 6 December 2019 / Accepted: 14 January 2020 / Published online: 30 January 2020

(c) The Author(s) 2020. This article is published with open access

\begin{abstract}
In POLLUX, daratumumab (D) plus lenalidomide/dexamethasone (Rd) reduced the risk of disease progression or death by $63 \%$ and increased the overall response rate (ORR) versus Rd in relapsed/refractory multiple myeloma (RRMM). Updated efficacy and safety after $>3$ years of follow-up are presented. Patients $(N=569)$ with $\geq 1$ prior line received Rd (lenalidomide, $25 \mathrm{mg}$, on Days 1-21 of each 28-day cycle; dexamethasone, $40 \mathrm{mg}$, weekly) \pm daratumumab at the approved dosing schedule. Minimal residual disease (MRD) was assessed by next-generation sequencing. After 44.3 months median follow-up, D-Rd prolonged progression-free survival (PFS) in the intent-to-treat population (median 44.5 vs 17.5 months; HR, 0.44; 95\% CI, 0.35-0.55; $P<0.0001)$ and in patient subgroups. D-Rd demonstrated higher ORR (92.9 vs $76.4 \%$; $P<0.0001)$ and deeper responses, including complete response or better $(56.6$ vs $23.2 \% ; P<0.0001)$ and MRD negativity $\left(10^{-5} ; 30.4\right.$ vs $\left.5.3 \% ; P<0.0001\right)$. Median time to next therapy was prolonged with D-Rd (50.6 vs 23.1 months; HR, 0.39 ; 95\% CI, 0.31-0.50; $P<0.0001)$. Median PFS on subsequent line of therapy (PFS2) was not reached with D-Rd versus 31.7 months with $\mathrm{Rd}(\mathrm{HR}, 0.53 ; 95 \% \mathrm{CI}, 0.42-0.68 ; P<0.0001)$. No new safety concerns were reported. These data support using D-Rd in patients with RRMM after first relapse.
\end{abstract}

Supplementary information The online version of this article (https:// doi.org/10.1038/s41375-020-0711-6) contains supplementary material, which is available to authorized users.

Nizar J. Bahlis

nbahlis@ucalgary.ca

1 University of Calgary, Charbonneau Cancer Research Institute, Calgary, AB, Canada

2 The National and Kapodistrian University of Athens, Athens, Greece

3 QEII Health Sciences Center and Dalhousie University, Halifax, NS, Canada

4 Service d'Hématologie et Thérapie Cellulaire, Hôpital Bretonneau, Centre Hospitalier Régional Universitaire (CHRU), Tours, France

5 St James's Institute of Oncology, Leeds Teaching Hospitals National Health Service Trust and University of Leeds, Leeds, UK

6 Assuta University Hospital, Faculty of Health Science, Ben-Gurion University of the Negev, Beersheba, Israel
Institute of Haematology, Royal Prince Alfred Hospital, Camperdown, NSW, Australia

8 Department of Medicine, Samsung Medical Center, Sungkyunkwan University School of Medicine, Seoul, South Korea

9 Department of Hematology, National Hospital Organization Disaster Medical Center of Japan, Tachikawa, Japan

10 Hematology, University Hospital Hôtel-Dieu, Nantes, France

11 Winship Cancer Institute, Emory University, Atlanta, GA, USA

12 Janssen Research \& Development, LLC, Spring House, PA, USA

13 Janssen Research \& Development, LLC, Raritan, NJ, USA

14 Janssen Global Medical Affairs, Horsham, PA, USA

15 Clínica Universidad de Navarra-Centro de Investigación Médica Aplicada, Instituto de Investigación Sanitaria de Navarra, Centro de Investigación Biomédica en Red de Cáncer, Pamplona, Spain 


\section{Introduction}

The development of first- and second-generation novel agents over the past decade has led to the acceptance of immunomodulatory drug (IMiD)-based or proteasome inhibitor (PI)-based doublet or triplet therapy as standard of care for newly diagnosed and relapsed or refractory multiple myeloma (RRMM) [1, 2]. However, multiple myeloma (MM) remains an incurable disease, underscoring the need for new treatment strategies. Optimal combination and sequencing of these next-generation agents remain to be defined, particularly among various patient subgroups.

Daratumumab is a human immunoglobulin Gк monoclonal antibody targeting CD38 with a direct on-tumor [3-6] and immunomodulatory mechanism of action [7-9]. Daratumumab-induced on-tumor activity occurs through several CD38 immune-mediated actions (complementdependent cytotoxicity, antibody-dependent cellular cytotoxicity, and antibody-dependent cellular phagocytosis), apoptosis, and modulation of CD38 enzymatic activity [3-6]. The immunomodulatory actions of daratumumab minimize the immune-suppressive functions of $\mathrm{CD} 8^{+}$myeloid-derived tumor suppressor cells, regulatory $\mathrm{T}$ cells, and regulatory $\mathrm{B}$ cells and increase T-cell clonality [7-9].

Daratumumab has demonstrated single-agent activity in heavily pretreated RRMM and in combination with standard-of-care regimens in RRMM after at least one prior therapy [10-13]. Daratumumab safety and efficacy are also established in combination with bortezomib, melphalan, and prednisone, and with lenalidomide and dexamethasone in patients with newly diagnosed MM (NDMM) who are transplantation ineligible [14, 15].

In the prespecified interim analysis of the phase 3 POLLUX study (median follow-up, 13.5 months), daratumumab in combination with lenalidomide and dexamethasone (D-Rd) reduced the risk of disease progression or death by $63 \%$ (median progression-free survival [PFS] not reached vs 18.4 months; hazard ratio [HR], 0.37; 95\% confidence interval [CI], 0.27-0.52; $P<$ 0.001 ) and significantly increased the overall response rate (ORR) compared with lenalidomide and dexamethasone $(\mathrm{Rd})$ alone (93 vs $76 \% ; P<0.001)$ in patients with at least one prior therapy [12]. In an updated analysis after a longer follow-up of 25.4 months, the PFS benefit of D-Rd was maintained compared with $\mathrm{Rd}$, with median PFS still not being reached for D-Rd versus 17.5 months for Rd. In addition, deep and durable responses were achieved with D-Rd, and the PFS benefit of D-Rd versus $\mathrm{Rd}$ was consistently maintained regardless of the number of prior lines of therapy received, prior IMiD exposure, bortezomib refractoriness, time since last therapy, or cytogenetic risk [16].
We report the long-term efficacy and safety analyses of POLLUX after a median follow-up of more than 3.5 years.

\section{Subjects and methods}

\section{Study design and patients}

POLLUX is an ongoing, randomized, open-label, multicenter, phase 3 study in patients with RRMM (ClinicalTrials.gov Identifier: NCT02076009). An independent ethics committee or institutional review board at each site approved the trial, and all patients provided written informed consent. The study protocol was conducted in accordance with the principles of the Declaration of Helsinki and the International Conference on Harmonisation Good Clinical Practice guidelines. The study design, primary results, and post hoc secondary analyses have been previously reported $[12,16]$. Briefly, eligible patients had progressive disease (according to International Myeloma Working Group [IMWG] criteria) [17, 18] during or after their last regimen, received and responded to at least one prior line of therapy, and had a creatinine clearance $\geq 30 \mathrm{~mL} / \mathrm{min}$. Prior lenalidomide exposure was allowed, but patients with lenalidomide-refractory disease were excluded from participation. A total of 569 patients were randomly assigned (1:1) using an interactive web response system to Rd (lenalidomide: $25 \mathrm{mg}$ orally on Days $1-21$ of each 28 day cycle; dexamethasone: $40 \mathrm{mg}$ orally weekly) with or without daratumumab $(16 \mathrm{mg} / \mathrm{kg}$ intravenous weekly for 8 weeks, every 2 weeks for 16 weeks, and every 4 weeks thereafter) until progression. The randomization was balanced by using randomly permuted blocks and was stratified by International Staging System (ISS), number of prior lines of therapy ( 1 vs 2 or 3 vs $>3$ ), and prior lenalidomide exposure. Treatment assignments were not blinded.

\section{Endpoints and assessments}

The primary efficacy endpoint was PFS. Secondary efficacy endpoints included ORR, rates of very good partial response (VGPR) or better and complete response (CR) or better, minimal residual disease (MRD), time to response, duration of response, and overall survival (OS). PFS on subsequent line of therapy (PFS2) was an exploratory endpoint and was defined as the time from randomization to progression after the next line of subsequent therapy or death.

Exploratory post hoc secondary analyses evaluated patient subgroups according to prior lines of therapy ( 1 and 1-3), prior lenalidomide treatment, refractoriness to bortezomib, and achievement of $\mathrm{CR}$ or better. The number of 
prior lines of therapy was determined by investigators according to the IMWG consensus guidelines [18]. PFS, ORR, and MRD negativity were assessed for each subgroup.

MRD was assessed at the time of suspected CR and at 3 and 6 months after confirmed CR (and every 12 months thereafter if $\mathrm{CR}$ was maintained) using clonoSEQ ${ }^{\circledR} \mathrm{V} 2.0$ (Adaptive Biotechnologies, Seattle, WA, USA). To allow for stringent, unbiased MRD evaluation, the entire intent-totreat (ITT) population was evaluated, and patients were considered MRD positive if they had MRD-positive test results or no MRD assessment.

\section{Statistical analyses}

Statistical analyses have been described previously [12]. Approximately 560 patients were randomized to observe 295 PFS events to detect an HR of 0.7 for the D-Rd group relative to the $\mathrm{Rd}$ group with $85 \%$ power at a two-sided significance level of 0.025 , using a group sequential testing design.

PFS was compared between treatment groups based on a stratified log-rank test. HRs and 95\% CIs were estimated using a stratified Cox regression model with treatment as the sole explanatory variable, and the Kaplan-Meier method was used to estimate the distributions. Stratified Cochran-Mantel-Haenszel tests were used to test treatment differences in ORRs and rates of VGPR or better and CR or better. MRD-negative rates were compared between groups using a Fisher's exact test and the likelihoodratio test.

\section{Results}

Between June 16, 2014 and July 14, 2015, 569 patients at 135 sites in 18 countries across North America, Europe, and the Asia Pacific region were randomly assigned in POLLUX; 286 patients were assigned to D-Rd and 283 patients were assigned to Rd. Baseline patient demographics, prior treatment history, and other clinical and cytogenetic characteristics have been previously published $[12,16]$ and are summarized in Supplementary Table 1. Approximately half of patients $(52 \%)$ had received one prior line of therapy, $18 \%$ had received prior lenalidomide, $44 \%$ had received prior IMiD and PI, and $21 \%$ were refractory to bortezomib.

At the clinical cutoff on October 10, 2018, a total of 158 (55.8\%) patients in the D-Rd group and 237 (84.3\%) patients in the Rd group had discontinued treatment. The most common reasons for discontinuation of treatment were progressive disease (D-Rd, 33.2\%; Rd, 59.4\%) and adverse events (AEs; D-Rd, 14.8\%; Rd, 14.9\%). The median (range) duration of study treatment was $34.3(0-50.8)$ months in the D-Rd group and $16.0(0.2-50.5)$ months in the $\mathrm{Rd}$ group.

\section{Efficacy}

For the primary endpoint, at a median (range) follow-up of 44.3 (0-50.9) months, D-Rd significantly prolonged PFS compared with Rd in the ITT population (median 44.5 [95\% CI, 34.1-not estimable] vs 17.5 [95\% CI, 13.9-20.8] months; HR, 0.44; 95\% CI, 0.35-0.55; $P<0.0001$; Fig. 1a). In the subgroup of patients who received one prior line of therapy, D-Rd ( $n=149)$ significantly prolonged PFS versus $\mathrm{Rd}(n=146$; median not reached vs 19.6 months; HR, 0.42; 95\% CI, 0.30-0.58; $P<0.0001$; Fig. 1b); 42-month PFS rates were $57.3 \%$ versus $27.8 \%$, respectively. Among patients who received one to three prior lines of therapy, D-Rd $(n=272)$ significantly prolonged PFS versus Rd ( $n=264$; median 44.5 vs 17.5 months; HR, 0.43 ; $95 \%$ CI, $0.34-0.54 ; P<0.0001)$. In patients who achieved deep responses of CR or better, PFS was prolonged with D-Rd $(n=159)$ versus $\mathrm{Rd}(n=64)$, with 42-month PFS rates of $73.6 \%$ versus $59.6 \%$, respectively; Fig. 1c. In patients with prior lenalidomide therapy, PFS was significantly prolonged with D-Rd $(n=50)$ versus $\mathrm{Rd}(n=50$; median 38.8 vs 18.6 months; HR, 0.38 ; 95\% CI, $0.21-0.66 ; P=0.0004$; Fig. 1d). In the subset of patients with bortezomibrefractory disease, PFS was significantly prolonged with D-Rd $(n=59)$ versus $\mathrm{Rd} \quad(n=58$; median 34.3 vs 11.3 months; HR, 0.40; 95\% CI, 0.24-0.67; $P=0.0003$; Fig. 1e). The PFS benefit of D-Rd versus Rd was also maintained in patients who received two or three lines of prior therapy, and in subgroups based on cytogenetic risk status, age, type of MM, ISS disease stage, Eastern Cooperative Oncology Group performance status score, baseline renal or hepatic function, prior treatment exposure, and refractory status (Fig. 2).

In the response-evaluable population, ORR was significantly higher with $\mathrm{D}-\mathrm{Rd}(n=281)$ compared with $\mathrm{Rd}$ alone $(n=276 ; 92.9$ vs $76.4 \% ; P<0.0001$; Table 1$)$, including higher rates of VGPR or better (80.4 vs $49.3 \%$; $P<0.0001)$ and CR or better (56.6 vs $23.2 \% ; P<0.0001$ ). Stringent CRs were achieved in $29.2 \%$ of patients in the D-Rd group versus $10.5 \%$ of patients in the $\mathrm{Rd}$ group. Among patients who received prior lenalidomide, ORR was significantly higher with D-Rd $(n=50)$ compared with $\mathrm{Rd}$ alone $(n=47 ; 84.0$ vs $64.0 \% ; P=0.0233$; Table 1$)$, including higher rates of VGPR or better ( 80.0 vs $36.0 \%$; $P<0.0001)$, CR or better (54.0 vs $12.0 \% ; P<0.0001)$, and stringent CR (26.0 vs $2.0 \%)$. At a sensitivity threshold of $10^{-5}$, MRD negativity was achieved by $87(30.4 \%)$ patients in the ITT population who received D-Rd versus 15 (5.3\%) patients who received $\mathrm{Rd}(P<0.0001)$. Among patients 

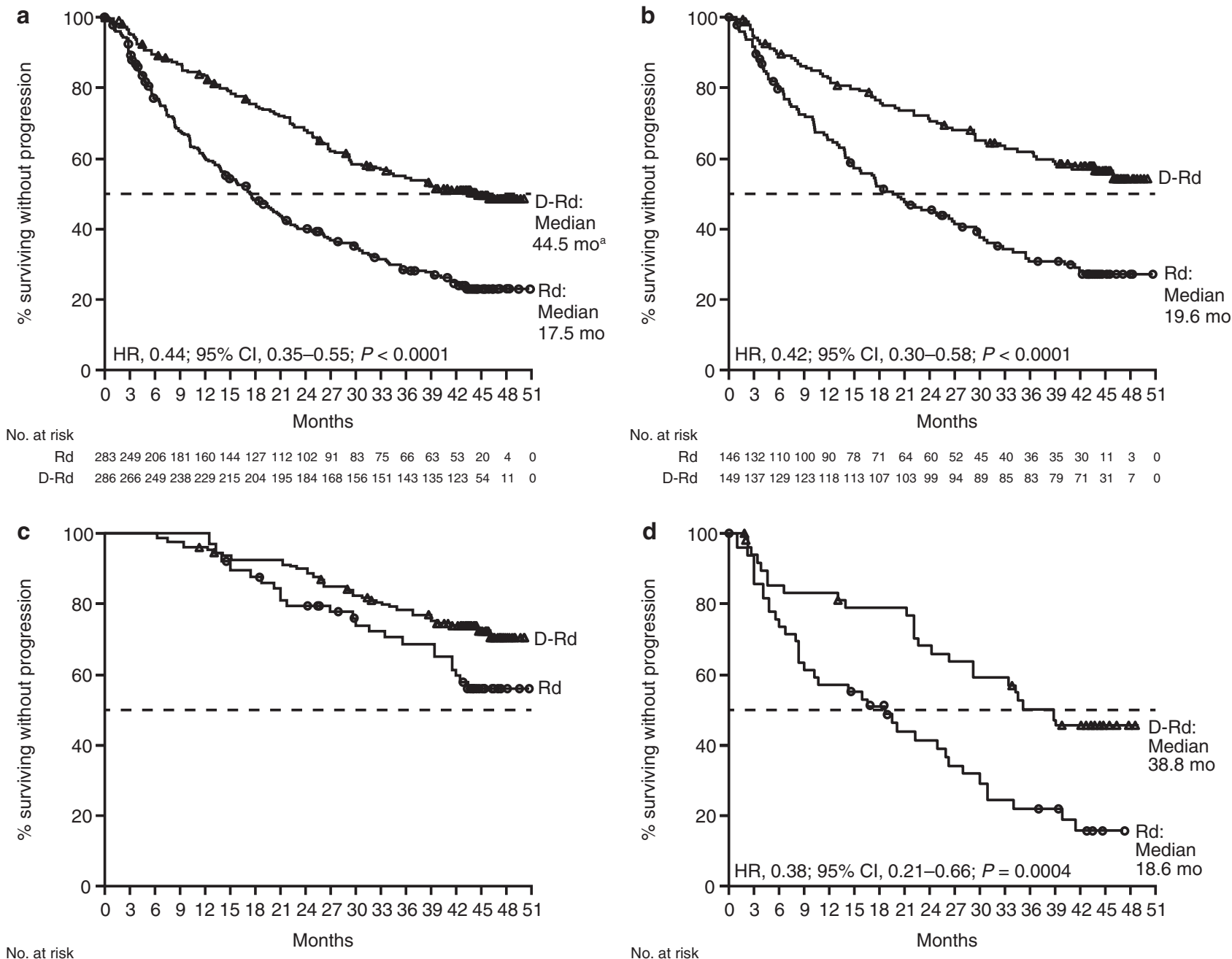

No. at risk

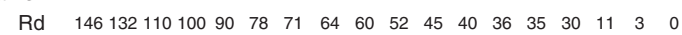

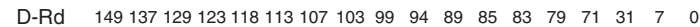

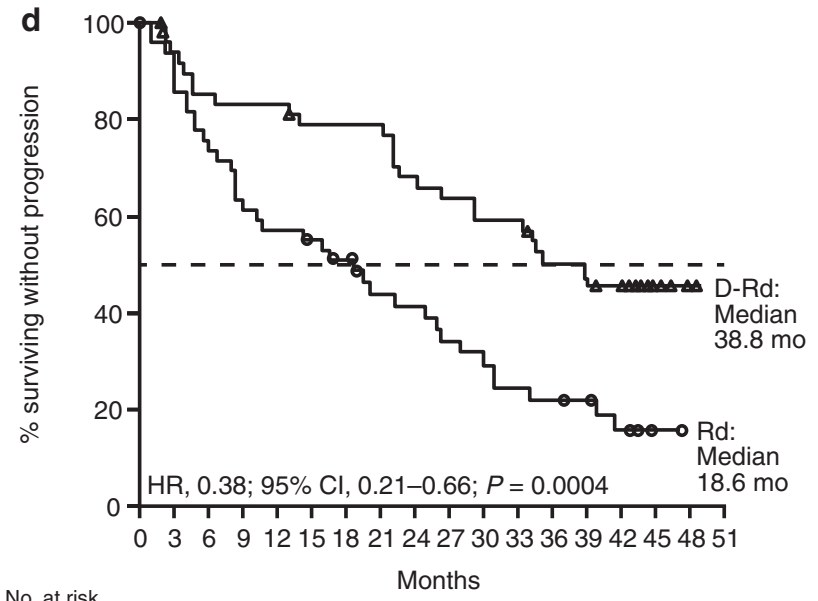

No. at risk

$\begin{array}{lllllllllllllllllll}\mathrm{Rd} & 50 & 43 & 37 & 31 & 28 & 26 & 23 & 18 & 17 & 14 & 12 & 10 & 9 & 8 & 5 & 1 & 0 & 0\end{array}$

$\begin{array}{lllllllllllllllllll}\mathrm{D}-\mathrm{Rd} & 50 & 44 & 40 & 39 & 39 & 36 & 36 & 36 & 31 & 29 & 27 & 27 & 22 & 20 & 19 & 5 & 1 & 0\end{array}$

$\begin{array}{lllllllllllllllllll}\mathrm{Rd} & 64 & 64 & 64 & 63 & 63 & 58 & 55 & 50 & 49 & 45 & 41 & 40 & 37 & 37 & 33 & 15 & 3 & 0\end{array}$

D-Rd $15915915915615114614514514113212712211911410444 \quad 8 \quad 0$

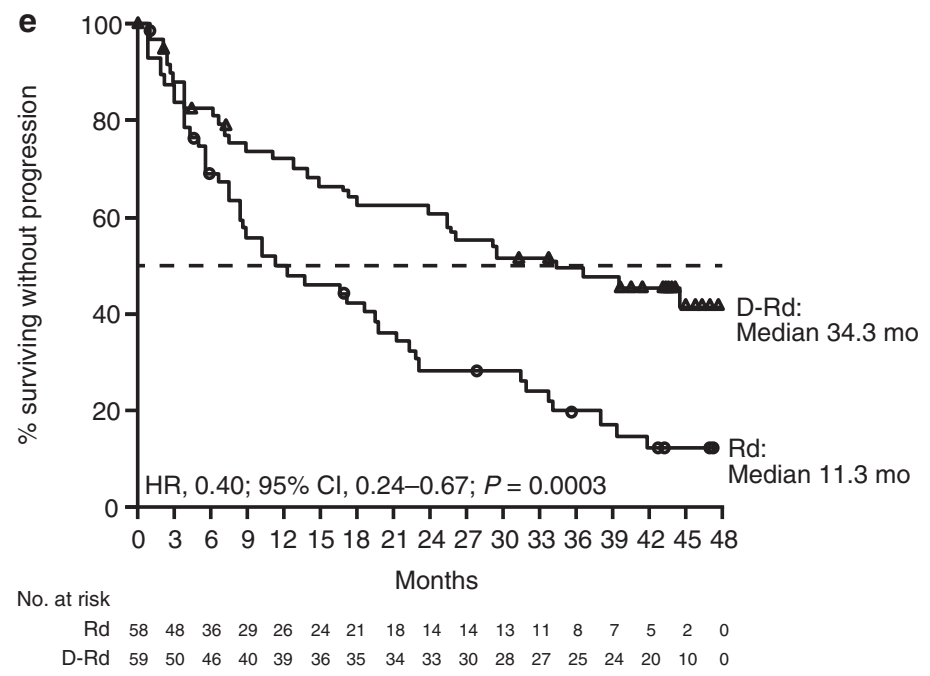

Fig. 1 PFS in the ITT population and in patient subgroups based on prior treatment. PFS in (a) the ITT population ${ }^{\mathrm{a}}$ and in patients with (b) one prior line of therapy, (c) responses of CR or better, (d) prior lenalidomide exposure, or (e) refractoriness to bortezomib. Kaplan-Meier estimates of PFS. PFS, progression-free survival;
ITT, intent-to-treat; D-Rd, daratumumab/lenalidomide/dexamethasone; $\mathrm{Rd}$, lenalidomide/dexamethasone; HR, hazard ratio; CI, confidence interval; NE, not estimable. ${ }^{\mathrm{a}}$ The upper bound of the $95 \%$ CI is currently NE. 


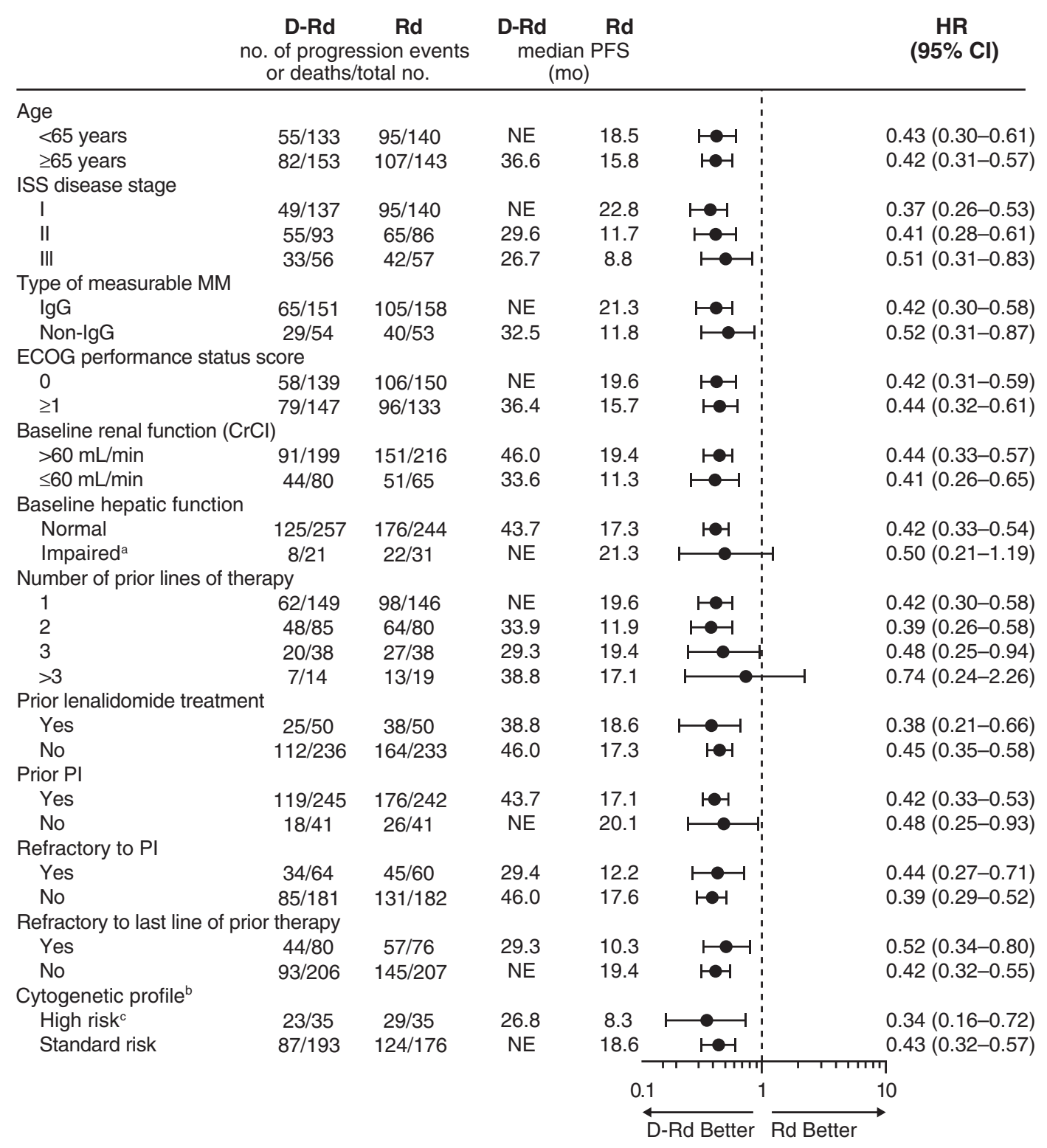

Fig. 2 PFS in patient subgroups. PFS, progression-free survival; D-Rd, daratumumab/lenalidomide/dexamethasone; Rd, lenalidomide/ dexamethasone; CI, confidence interval; NE, not estimable; ISS, International Staging System; MM, multiple myeloma; Ig, immunoglobulin; ECOG, Eastern Cooperative Oncology Group; $\mathrm{CrCl}$, creatinine clearance; PI, proteasome inhibitor; FISH, fluorescence

who achieved MRD negativity $\left(10^{-5}\right)$, PFS was prolonged with D-Rd versus Rd (median not reached vs 42.0 months; HR, 0.46; 95\% CI, 0.19-1.08; $P=0.0667$; Fig. 3), with 42-month PFS rates of $76.7 \%$ with D-Rd and $42.8 \%$ with Rd. Among patients with MRD-positive status, D-Rd significantly prolonged PFS compared with Rd (median 29.4 vs 16.0 months; HR, 0.60; 95\% CI, 0.48-0.76; $P<0.0001$; Fig. 3). Among patients who received prior lenalidomide, a similar improvement in the rate of MRD negativity $\left(10^{-5}\right)$ was observed with D-Rd (32.0\%) versus $\mathrm{Rd}$ alone $(6.0 \%$; $P=0.0006)$.

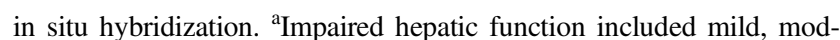
erate, and severe hepatic dysfunction as per National Cancer Institute organ dysfunction criteria. ${ }^{\mathrm{b}}$ Cytogenetic risk was determined by FISH or karyotyping. ${ }^{c}$ Patients with high cytogenetic risk had $t(4 ; 14)$, $t(14 ; 16)$, or del17p abnormalities.

Median duration of response was not reached $(95 \% \mathrm{CI}$, could not be estimated) with D-Rd compared with 25.2 (95\% CI, 19.3-29.7) months with Rd. Median time to next therapy was 50.6 months versus 23.1 months in the D-Rd and Rd arms, respectively (HR, 0.39; 95\% CI, 0.31-0.50; $P<0.0001$; Fig. 4a). A total of 63 patients in the Rd group received daratumumab monotherapy after disease progression on Rd. At the time of this analysis, seven patients were on subsequent daratumumab monotherapy. The most common reason for discontinuation of daratumumab monotherapy was disease progression. Median PFS2 was not 
Table 1 Summary of best confirmed response ${ }^{a}$ and MRD-negative ${ }^{b}$ rates.

\begin{tabular}{llll}
\hline Variable & $\mathrm{D}-\mathrm{Rd}(n=281)$ & $\mathrm{Rd}(n=276)$ & $P$ \\
\hline Overall response & & & \\
No. with response & 261 & 211 & $<0.0001^{\mathrm{c}}$ \\
Rate, \% (95\% CI) & 92.9 & 76.4 & \\
& $(89.2-95.6)$ & $(71.0-81.3)$ & \\
Clinical benefit, $n(\%)^{\mathrm{d}}$ & $266(94.7)$ & $237(85.9)$ & \\
Best overall response, $n(\%)$ & & \\
CR or better & $159(56.6)$ & $64(23.2)$ & $<0.0001^{\mathrm{c}}$ \\
$\quad$ Stringent CR & $82(29.2)$ & $29(10.5)$ & \\
$\quad$ CR & $77(27.4)$ & $35(12.7)$ & \\
VGPR or better & $226(80.4)$ & $136(49.3)$ & $<0.0001^{\mathrm{c}}$ \\
VGPR & $67(23.8)$ & $72(26.1)$ & \\
Partial response & $35(12.5)$ & $75(27.2)$ & \\
Stable disease & $18(6.4)$ & $59(21.4)$ & \\
Progressive disease & $0(0.0)$ & $4(1.4)$ & \\
Response could not & $2(0.7)$ & $2(0.7)$ & \\
be evaluated & & & \\
MRD negative $\left(10^{-5}\right)$ & $n=286$ & $n=283$ & \\
$n(\%)$ & $87(30.4)$ & $15(5.3)$ & \\
\hline Response was assessed &
\end{tabular}

Response was assessed according to the Uniform Criteria Consensus recommendations of the International Myeloma Working Group $[17,18]$. The analysis included patients who had a confirmed diagnosis of $\mathrm{MM}$ and measurable disease at baseline or screening. In addition, patients had received at least one administration of trial treatment and had at least one disease assessment after the baseline visit.

$M R D$ minimal residual disease, $D-R d$ daratumumab/lenalidomide/ dexamethasone, $R d$ lenalidomide/dexamethasone, $C I$ confidence interval, $C R$ complete response, $V G P R$ very good partial response.

${ }^{a}$ Response-evaluable population.

bintent-to-treat population.

${ }^{c} P$ value was calculated using the Cochran-Mantel-Haenszel chisquare test.

${ }^{\mathrm{d}}$ Clinical benefit includes all patients with minimal response, partial response, VGPR, CR, and stringent $\mathrm{CR}$.

${ }^{\mathrm{e}}$ Criteria for a stringent $\mathrm{CR}$ include the criteria for a $\mathrm{CR}$ plus a normal free light-chain ratio and the absence of clonal plasma cells as assessed by immunohistochemical or immunofluorescence analysis or by flow cytometry.

${ }^{\mathrm{f}}$ Includes patients who achieved a minimal response.

${ }^{\mathrm{g}} P$ value was calculated using the Fisher's exact test.

reached in the D-Rd group versus 31.7 months in the $\mathrm{Rd}$ group (HR, 0.53; 95\% CI, 0.42-0.68; $P<0.0001$; Fig. 4b), with 42-month PFS2 rates of $59 \%$ and $38 \%$, respectively. At the time of this analysis, fewer deaths occurred in patients receiving D-Rd $(n=104)$ compared with $\mathrm{Rd}(n=$ 121 ), and median OS was not reached in either group. The 42 -month OS rate was $65 \%$ with D-Rd versus $57 \%$ with Rd. Follow-up for OS is ongoing.

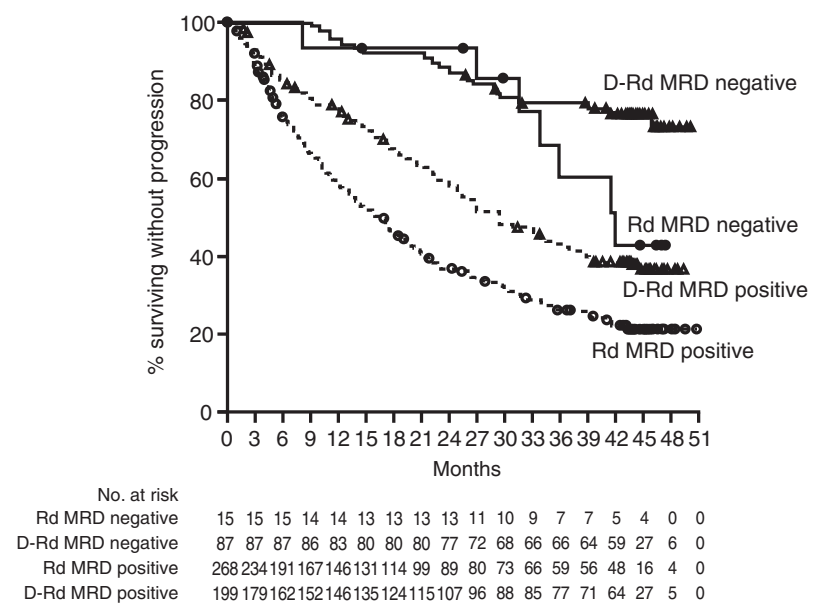

Fig. 3 PFS based on MRD status $\left(\mathbf{1 0}^{-5}\right)$. PFS, progression-free survival; MRD, minimal residual disease; D-Rd, daratumumab/lenalidomide/dexamethasone; Rd, lenalidomide/dexamethasone.

\section{Safety}

No new safety concerns were reported in either treatment group with longer follow-up. The most common treatmentemergent AE (TEAE) was neutropenia, occurring in 63.3\% of patients treated with D-Rd and $48.0 \%$ of patients who received $\mathrm{Rd}$ (Table 2 ). The most common ( $\geq 5 \%$ ) grade $3 / 4$ TEAEs observed with D-Rd and Rd included neutropenia, febrile neutropenia, anemia, thrombocytopenia, lymphopenia, pneumonia, diarrhea, fatigue, hypokalemia, and cataracts (Table 2). The percentage of patients with TEAEs leading to treatment discontinuation was similar between groups (D-Rd, 14.8; Rd, 14.6\%). The most common TEAEs $(\geq 1 \%)$ leading to treatment discontinuation with D-Rd versus $\mathrm{Rd}$ were pneumonia ( 1.8 vs $1.1 \%)$, pulmonary embolism (0 vs $1.1 \%$ ), septic shock (1.1 vs $0 \%$ ), and general physical health deterioration (1.1 vs $0 \%)$, respectively. The incidence of second primary malignancies was similar between groups, occurring in $8.5 \%$ of patients who received D-Rd and $8.9 \%$ of patients who received Rd (Table 3).

\section{Discussion}

After $>3.5$ years of median follow-up, the addition of daratumumab to $\mathrm{Rd}$ continued to demonstrate significant clinical benefit over Rd alone in patients with RRMM. At a median follow-up of 44.3 months, D-Rd demonstrated an unprecedented median PFS of 44.5 months versus only 17.5 months for $\mathrm{Rd}$, conferring a $56 \%$ reduction in the risk of disease progression or death. At the time of the analysis, the upper bound of the $95 \% \mathrm{CI}$ for median PFS in the D-Rd group was not estimable. Deep responses, including significantly higher ( $>5$-fold) rates of MRD negativity $\left(10^{-5}\right)$ 
a

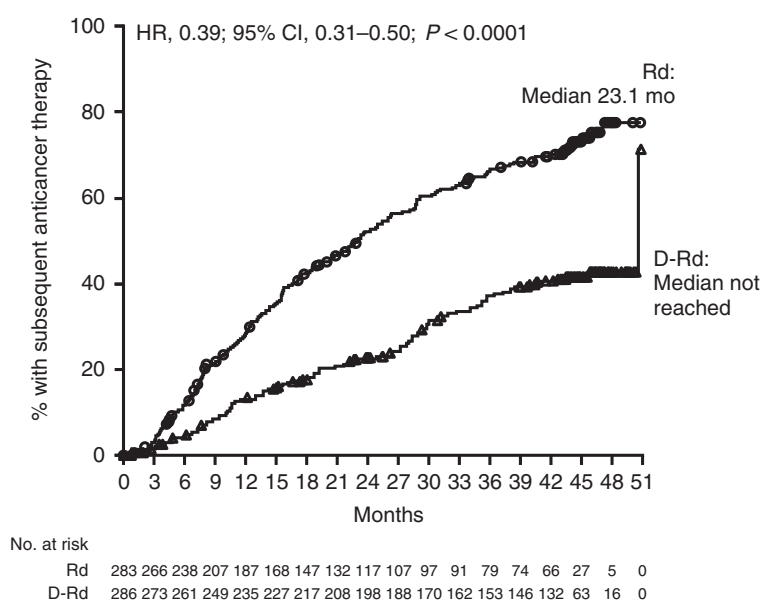

Fig. 4 Time to subsequent therapy and PFS2. Time to subsequent therapy (a) and PFS2 (b) in the ITT population. PFS2, progressionfree survival on subsequent line of therapy; HR, hazard ratio;

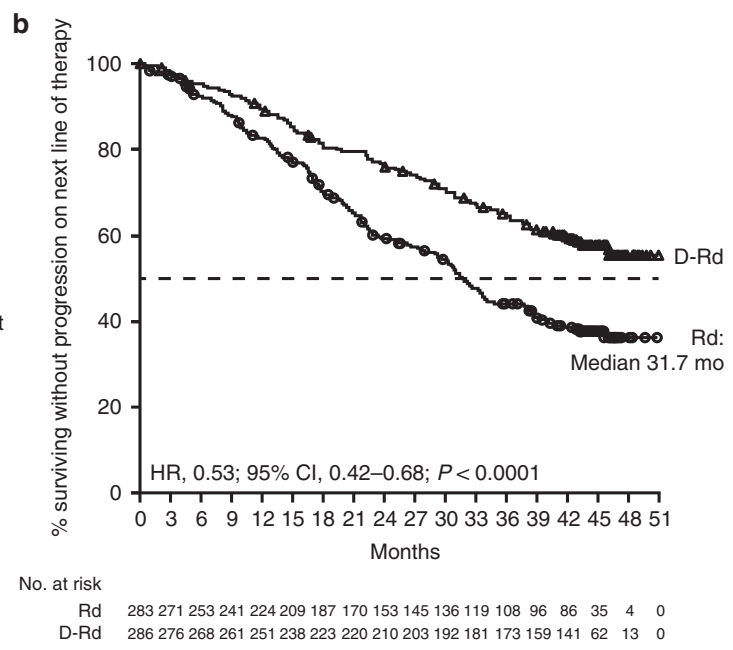

CI, confidence interval; Rd, lenalidomide/dexamethasone; D-Rd, daratumumab/lenalidomide/dexamethasone; ITT, intent-to-treat.

48.8 months for KRd and 48.0 months for Rd [20]. With longer follow-up (median 67.1 months), median OS was 48.3 months for KRd versus 40.4 months for $\mathrm{Rd}$, resulting in $21 \%$ reduction in the risk of death $(\mathrm{HR}, 0.79 ; 95 \% \mathrm{CI}$, 0.67-0.95; $P=0.0045) \quad$ [20]. In the phase 3 TOURMALINE-MM1 study in patients with RRMM and one to three prior therapies, median PFS was 20.6 months with ixazomib in combination with $\mathrm{Rd}$ (IRd) versus 14.7 months with Rd alone (HR, 0.74; 95\% CI, 0.59-0.94; $P=0.01)$, at a median follow-up of 14.8 versus 14.6 months in the IRd and Rd groups, respectively [21]. Median PFS in the phase 3 ELOQUENT-2 study in patients with RRMM and one to three prior therapies was 19.4 months with elotuzumab plus $\mathrm{Rd}$ compared with 14.9 months with Rd alone (HR, 0.73; 95\% CI, 0.60-0.89; $P=0.0014$ ), with 3 years of extended follow-up [22].

D-Rd induced deep and durable responses that continued to deepen over time with longer follow-up. At the most recent clinical cutoff, rates of VGPR or better (80.4 vs $49.3 \%$ ) and CR or better (56.6 vs $23.2 \%$ ) with D-Rd versus $\mathrm{Rd}$ were higher than those observed at primary analysis (VGPR or better: 75.8 vs $44.2 \%$; CR or better: 43.1 vs $19.2 \%$ ) [12] and at $\sim 2$ years of follow-up (VGPR or better: 78.6 vs $47.8 \%$; CR or better: 51.2 vs $21.0 \%$; Supplementary Fig. 1a) [16]. MRD-negative rates have also continued to deepen with $\mathrm{D}-\mathrm{Rd}$ versus $\mathrm{Rd}$ over time (interim analysis: 22.4 vs $4.6 \%$ [12]; 2-year follow-up: 26.2 vs $6.4 \%$ [16]; current analysis: 30.4 vs 5.3\%; Supplementary Fig. 1b), while the rates of MRD negativity with $\mathrm{Rd}$ have remained relatively constant with longer follow-up. It is important to note that an updated next-generation sequencing assay with improved calibration rate was used to determine MRD negativity in the current study. PFS was prolonged with $0.55-0.78 ; \quad P<0.001)$, at a median follow-up of 
Table 2 Most common all grade $(\geq 25 \%)$ and grade $3 / 4(\geq 5 \%)$

TEAEs in the safety population.

\begin{tabular}{|c|c|c|c|c|}
\hline \multirow[t]{2}{*}{ Event } & \multicolumn{2}{|l|}{$\mathrm{D}-\mathrm{Rd}(n=283)$} & \multicolumn{2}{|l|}{$\operatorname{Rd}(n=281)$} \\
\hline & All grade, $n(\%)$ & Grade $3 / 4, n(\%)$ & All grade, $n(\%)$ & Grade $3 / 4, n(\%)$ \\
\hline Total & $281(99.3)$ & $255(90.1)$ & $274(97.5)$ & $227(80.8)$ \\
\hline \multicolumn{5}{|l|}{ Hematologic } \\
\hline Neutropenia & $179(63.3)$ & $157(55.5)$ & $135(48.0)$ & 117 (41.6) \\
\hline Febrile neutropenia & $18(6.4)$ & $18(6.4)$ & $8(2.8)$ & $8(2.8)$ \\
\hline Anemia & $111(39.2)$ & $50(17.7)$ & $114(40.6)$ & $60(21.4)$ \\
\hline Thrombocytopenia & $87(30.7)$ & $42(14.8)$ & $88(31.3)$ & $44(15.7)$ \\
\hline Lymphopenia & $19(6.7)$ & $16(5.7)$ & $17(6.0)$ & $12(4.3)$ \\
\hline \multicolumn{5}{|l|}{ Nonhematologic } \\
\hline Diarrhea & $165(58.3)$ & $28(9.9)$ & $105(37.4)$ & $11(3.9)$ \\
\hline $\begin{array}{l}\text { Upper respiratory tract } \\
\text { infection }\end{array}$ & $121(42.8)$ & $5(1.8)$ & $78(27.8)$ & $5(1.8)$ \\
\hline Fatigue & $110(38.9)$ & $19(6.7)$ & $87(31.0)$ & $12(4.3)$ \\
\hline Cough & $99(35.0)$ & $1(0.4)$ & $42(14.9)$ & $0(0.0)$ \\
\hline Nasopharyngitis & $96(33.9)$ & $0(0.0)$ & $59(21.0)$ & $0(0.0)$ \\
\hline Constipation & $93(32.9)$ & $3(1.1)$ & $76(27.0)$ & $2(0.7)$ \\
\hline Muscle spasms & $84(29.7)$ & $3(1.1)$ & $60(21.4)$ & $4(1.4)$ \\
\hline Nausea & $82(29.0)$ & $6(2.1)$ & $51(18.1)$ & $2(0.7)$ \\
\hline Insomnia & $76(26.9)$ & $6(2.1)$ & $63(22.4)$ & $4(1.4)$ \\
\hline Pyrexia & $73(25.8)$ & $9(3.2)$ & $40(14.2)$ & $7(2.5)$ \\
\hline Back pain & $71(25.1)$ & $8(2.8)$ & $57(20.3)$ & $5(1.8)$ \\
\hline Pneumonia & $71(25.1)$ & $43(15.2)$ & $46(16.4)$ & $28(10.0)$ \\
\hline Edema peripheral & 67 (23.7) & $2(0.7)$ & 47 (16.7) & $4(1.4)$ \\
\hline Vomiting & 62 (21.9) & $3(1.1)$ & $19(6.8)$ & $4(1.4)$ \\
\hline Dyspnea & 61 (21.6) & $12(4.2)$ & $37(13.2)$ & $2(0.7)$ \\
\hline Bronchitis & $57(20.1)$ & $7(2.5)$ & $48(17.1)$ & $9(3.2)$ \\
\hline Asthenia & $54(19.1)$ & $10(3.5)$ & $46(16.4)$ & $9(3.2)$ \\
\hline Cataract & $54(19.1)$ & $17(6.0)$ & 33 (11.7) & $12(4.3)$ \\
\hline Hypokalemia & $51(18.0)$ & $17(6.0)$ & $31(11.0)$ & $9(3.2)$ \\
\hline Headache & $49(17.3)$ & $0(0.0)$ & $24(8.5)$ & $0(0.0)$ \\
\hline
\end{tabular}

$T E A E$ treatment-emergent adverse event, $D$-Rd daratumumab/lenalidomide/dexamethasone, $R d$ lenalidomide/dexamethasone.
Table 3 Summary of second primary malignancies in the safety population.

\begin{tabular}{lcc}
\hline & $\mathrm{D}-\mathrm{Rd}(n=283)$ & $\mathrm{Rd}(n=281)$ \\
\hline Total, $n(\%)$ & $24(8.5)$ & $25(8.9)$ \\
Cutaneous/noninvasive & $12(4.2)$ & $10(3.6)$ \\
Noncutaneous/invasive & $8(2.8)$ & $11(3.9)$ \\
Hematologic & $5(1.8)$ & $3(1.1)$ \\
\hline
\end{tabular}

$D-R d$ daratumumab/lenalidomide/dexamethasone, $R d$ lenalidomide/ dexamethasone.

D-Rd versus Rd in patients who achieved MRD negativity and significantly prolonged with D-Rd versus Rd in patients with MRD-positive status. The lack of a statistically significant difference in PFS among patients who achieved MRD negativity with $\mathrm{D}-\mathrm{Rd}$ versus $\mathrm{Rd}(P=0.0667)$ may be the result of the low number of patients in the $\mathrm{Rd}$ arm $(n=15)$.

PFS2 may serve as a surrogate endpoint for OS when survival data are not available [23]. The use of PFS2 has been suggested as a preferred endpoint, particularly for studies investigating long-term maintenance treatment [23-25]. The findings presented here demonstrate that D-Rd significantly prolongs the time to subsequent therapy and PFS2 versus Rd, conferring a $47 \%$ reduction in the risk of disease progression or death on the next line of therapy. These data indicate that treatment with D-Rd does not negatively impact on patient outcomes on subsequent therapy. At the time of this analysis, 17 more deaths occurred with Rd (121/283) compared with D-Rd (104/ 286). Follow-up for OS in POLLUX is ongoing, with the final analysis planned after 330 deaths. 
With longer follow-up, the safety profile of D-Rd and Rd remains largely consistent with the known safety profiles of daratumumab [26] and of $\operatorname{Rd}[20,27,28]$. Despite the higher rates of neutropenia and infections (upper respiratory tract infection and pneumonia), the rates of grade 3 or 4 infections were similar between treatment groups and were managed according to local institutional treatment standardof-care protocols. Consistent tolerability was also observed with daratumumab in the phase 3 MAIA study of D-Rd versus Rd in patients with NDMM who are ineligible for transplantation (median follow-up, 28 months) [15].

Taken together, the results from $>3.5$ years of median follow-up demonstrate that D-Rd continues to provide significant PFS benefit and induces deeper and more durable responses, including a greater than five-fold increase in the rate of MRD negativity versus $\mathrm{Rd}$ alone in patients with RRMM. No new safety concerns were observed following a median of 34 months of D-Rd exposure. These updated findings continue to support the use of D-Rd in patients with RRMM after first relapse.

Acknowledgements This study was sponsored by Janssen Research \& Development, LLC. The authors thank the patients who participated in this study and their families, as well as the study co-investigators, research nurses, and coordinators at each of the clinical sites. Medical writing and editorial support were provided by Kristin Runkle, $\mathrm{PhD}$, of MedErgy and were funded by Janssen Global Services, LLC. The data sharing policy of Janssen Pharmaceutical Companies of Johnson \& Johnson is available at https://www.janssen.com/clinical-trials/transpa rency. As noted on this site, requests for access to the study data can be submitted through Yale Open Data Access (YODA) Project site at http://yoda.yale.edu.

Author contributions NJB, MAD, GC, ST, and JS-M conceived and designed the study, acquired data, and analyzed and interpreted results; DJW, LB, KK, NT, and LO acquired data; ML, PJH, JLK, and CC acquired data and analyzed and interpreted results; PM conceived and designed the study; MK and XQ analyzed and interpreted results; and $\mathrm{JU}$ and MQ conceived and designed the study and analyzed and interpreted results. All authors drafted and reviewed the manuscript, approved the final version, agreed to publish this report, and vouch for accuracy and completeness of the data.

\section{Compliance with ethical standards}

Conflict of interest NJB consulted for and received honoraria and research funding from Janssen, Celgene, and Amgen. MAD consulted for and received honoraria from Janssen, Celgene, Takeda, Amgen, and Bristol-Myers Squibb. DJW received honoraria from and served on advisory committees for Amgen, Celgene, Janssen, and Takeda. GC received honoraria from Amgen, Bristol-Myers Squibb, GlycoMimetics, Celgene, Janssen, Takeda, and Sanofi; and received research funding from Celgene, Janssen, and Takeda. PJH received honoraria from Janssen, Amgen, Novartis, and Takeda; and received travel support from Takeda and Celgene. PM received honoraria from and served on advisory committees for Amgen, Celgene, Janssen, Takeda, and AbbVie. JLK served in a consulting or advisory role for Janssen, Celgene, Takeda, Amgen, and Sanofi Genzyme; and received research funding from Janssen, Bluebird, Bristol-Myers Squibb, Celgene, and
Sutro Biopharma. MK, CC, XQ, LO, ST, JU, and MQ are employees of Janssen. JS-M received honoraria from Janssen, Celgene, Amgen, Bristol-Myers Squibb, Novartis, Sanofi, and Roche. LB, ML, KK, and NT have no conflicts of interest to disclose.

Publisher's note Springer Nature remains neutral with regard to jurisdictional claims in published maps and institutional affiliations.

Open Access This article is licensed under a Creative Commons Attribution 4.0 International License, which permits use, sharing, adaptation, distribution and reproduction in any medium or format, as long as you give appropriate credit to the original author(s) and the source, provide a link to the Creative Commons license, and indicate if changes were made. The images or other third party material in this article are included in the article's Creative Commons license, unless indicated otherwise in a credit line to the material. If material is not included in the article's Creative Commons license and your intended use is not permitted by statutory regulation or exceeds the permitted use, you will need to obtain permission directly from the copyright holder. To view a copy of this license, visit http://creativecommons. org/licenses/by/4.0/.

\section{References}

1. National Comprehensive Cancer Network. NCCN Clinical Practice Guidelines in Oncology ${ }^{\mathrm{TM}}$. Multiple Myeloma. Version 1.2019. 2018. https://www.nccn.org/professionals/physician_gls/ pdf/myeloma.pdf. Accessed 19 Oct 2018.

2. Moreau P, San Miguel J, Sonneveld P, Mateos MV, Zamagni E, Avet-Loiseau H, et al. Multiple myeloma: ESMO Clinical Practice Guidelines for diagnosis, treatment and follow-up. Ann Oncol. 2017;28(suppl_4):iv52-iv61.

3. de Weers M, Tai YT, van der Veer MS, Bakker JM, Vink T, Jacobs DC, et al. Daratumumab, a novel therapeutic human CD38 monoclonal antibody, induces killing of multiple myeloma and other hematological tumors. J Immunol. 2011;186:1840-8.

4. Lammerts van Bueren J, Jakobs D, Kaldenhoven N, Roza M, Hiddingh S, Meesters J, et al. Direct in vitro comparison of daratumumab with surrogate analogs of CD38 antibodies MOR03087, SAR650984 and Ab79. Blood. 2014;124:3474.

5. Overdijk MB, Verploegen S, Bogels M, van Egmond M, Lammerts van Bueren JJ, Mutis T, et al. Antibody-mediated phagocytosis contributes to the anti-tumor activity of the therapeutic antibody daratumumab in lymphoma and multiple myeloma. MAbs. 2015;7:311-21.

6. Overdijk MB, Jansen JH, Nederend M, Lammerts van Bueren JJ, Groen RW, Parren PW, et al. The therapeutic CD38 monoclonal antibody daratumumab induces programmed cell death via Fc gamma receptor-mediated cross-linking. J Immunol. 2016; 197:807-13.

7. Krejcik J, Casneuf T, Nijhof IS, Verbist B, Bald J, Plesner T, et al. Daratumumab depletes $\mathrm{CD}_{3} 8^{+}$immune-regulatory cells, promotes T-cell expansion, and skews T-cell repertoire in multiple myeloma. Blood. 2016;128:384-94.

8. Chiu C, Casneuf T, Axel A, Lysaght A, Bald J, Khokhar NZ, et al. Daratumumab in combination with lenalidomide plus dexamethasone induces clonality increase and T-cell expansion: results from a phase 3 randomized study (POLLUX). Blood. 2016; 128:4531.

9. Adams HC 3rd, Stevenaert F, Krejcik J, Van der Borght K, Smets $\mathrm{T}$, Bald J, et al. High-parameter mass cytometry evaluation of relapsed/refractory multiple myeloma patients treated with daratumumab demonstrates immune modulation as a novel mechanism of action. Cytometry A. 2019;95:279-89. 
10. Usmani SZ, Weiss BM, Plesner T, Bahlis NJ, Belch A, Lonial S, et al. Clinical efficacy of daratumumab monotherapy in patients with heavily pretreated relapsed or refractory multiple myeloma. Blood. 2016;128:37-44.

11. Palumbo A, Chanan-Khan A, Weisel K, Nooka AK, Masszi T, Beksac M, et al. Daratumumab, bortezomib, and dexamethasone for multiple myeloma. N Engl J Med. 2016;375:754-66.

12. Dimopoulos MA, Oriol A, Nahi H, San-Miguel J, Bahlis N, Usmani S, et al. Daratumumab, lenalidomide, and dexamethasone for multiple myeloma. N Engl J Med. 2016;375:1319-31.

13. Chari A, Suvannasankha A, Fay JW, Arnulf B, Kaufman JL, Ifthikharuddin JJ, et al. Daratumumab plus pomalidomide and dexamethasone in relapsed and/or refractory multiple myeloma. Blood. 2017;130:974-81.

14. Mateos MV, Dimopoulos MA, Cavo M, Suzuki K, Jakubowiak A, Knop S, et al. Daratumumab plus bortezomib, melphalan, and prednisone for untreated myeloma. N Engl J Med. 2018; 378:518-28.

15. Facon T, Kumar S, Plesner T, Orlowski RZ, Moreau P, Bahlis N, et al. Daratumumab plus lenalidomide and dexamethasone for untreated myeloma. N Engl J Med. 2019;380:2104-15.

16. Dimopoulos M, San Miguel J, Belch A, White D, Benboubker L, Cook G, et al. Daratumumab plus lenalidomide and dexamethasone versus lenalidomide and dexamethasone in relapsed or refractory multiple myeloma: updated analysis of POLLUX. Haematologica. 2018;103:2088-96.

17. Durie BGM, Harousseau JL, Miguel JS, Blade J, Barlogie B, Anderson $\mathrm{K}$, et al. International uniform response criteria for multiple myeloma. Leukemia. 2006;20:1467-73.

18. Rajkumar SV, Harousseau JL, Durie B, Anderson KC, Dimopoulos M, Kyle R, et al. Consensus recommendations for the uniform reporting of clinical trials: report of the International Myeloma Workshop Consensus Panel 1. Blood. 2011;117: 4691-5.

19. Mateos M-V, Sonneveld P, Hungria VTM, Nooka AK, Estell J, Barreto WG, et al. Efficacy and safety of daratumumab, bortezomib, and dexamethasone (D-Vd) versus bortezomib and dexamethasone $(\mathrm{Vd})$ in first relapse patients: two-year update of CASTOR. Poster presented at the 60th annual meeting of the American Society of Hematology; December 1-4, 2018; San Diego, CA. Abstract 3270.
20. Siegel DS, Dimopoulos MA, Ludwig H, Facon T, Goldschmidt H, Jakubowiak A, et al. Improvement in overall survival with carfilzomib, lenalidomide, and dexamethasone in patients with relapsed or refractory multiple myeloma. J Clin Oncol. 2018;36:728-34.

21. Moreau P, Masszi T, Grzasko N, Bahlis NJ, Hansson M, Pour L, et al. Oral ixazomib, lenalidomide, and dexamethasone for multiple myeloma. N Engl J Med. 2016;374:1621-34.

22. Dimopoulos MA, Lonial S, White D, Moreau P, Palumbo A, SanMiguel J, et al. Elotuzumab plus lenalidomide/dexamethasone for relapsed or refractory multiple myeloma: ELOQUENT-2 followup and post-hoc analyses on progression-free survival and tumour growth. Br J Haematol. 2017;178:896-905.

23. Oronsky B, Carter CA, Reid TR, Scicinski J, Oronsky A, Lybeck $\mathrm{M}$, et al. Confirmatory trials in the evaluation of anticancer medicinal products in man-PFS2: a measure of therapeutic action-at-a-distance. Neoplasia. 2015;17:716-22.

24. Solaman A, Walker AJ, Davies FE. Progression-free survival 2 in hematological cancer treatment choices: challenges to routine use. Blood. 2016;128:5982.

25. European Medicines Agency, Science Medicines Health. Guideline on the evaluation of anticancer medicinal products in man. 2012. Accessed 17 September 2019. https://www.ema.europa.eu/ en/documents/scientific-guideline/guideline-evaluation-antica ncer-medicinal-products-man-revision-4_en.pdf.

26. Usmani S, Nahi H, Weiss BM, Bahlis N, Belch A, Lokhorst $\mathrm{HM}$, et al. Safety and efficacy of daratumumab monotherapy in patients with heavily pretreated relapsed and refractory multiple myeloma: final results from GEN501 and SIRIUS. Poster presented at the 59th American Society of Hematology (ASH) annual meeting \& exposition; December 9-12, 2017; Atlanta, GA. Abstract 3107.

27. Dimopoulos MA, Swern AS, Li JS, Hussein M, Weiss L, Nagarwala Y, et al. Efficacy and safety of long-term treatment with lenalidomide and dexamethasone in patients with relapsed/ refractory multiple myeloma. Blood Cancer J. 2014;4:e257.

28. Dimopoulos MA, Lonial S, Betts KA, Chen C, Zichlin ML, Brun A, et al. Elotuzumab plus lenalidomide and dexamethasone in relapsed/refractory multiple myeloma: extended 4-year follow-up and analysis of relative progression-free survival from the randomized ELOQUENT-2 trial. Cancer. 2018;124:4032-43. 